\title{
Few-photons model of the optical emission of semiconductor quantum dots
}

\author{
Marten Richter,, , Alexander Carmele, ${ }^{1}$ Anna Sitek, ${ }^{1,2}$ and Andreas Knorr ${ }^{1}$ \\ ${ }^{1}$ Institut für Theoretische Physik, Nichtlineare Optik und Quantenelektronik, \\ Technische Universität Berlin, Hardenbergstr. 36, 10623 Berlin, Germany \\ ${ }^{2}$ Institute of Physics, Wroctaw University of Technology, \\ Wybrzeże Wyspiańskiego 27, 50-370 Wroctaw, Poland
}

\begin{abstract}
The Jaynes-Cummings model provides a well established theoretical framework for single electron two level systems in a radiation field. Similar exactly solvable models for semiconductor light emitters such as quantum dots dominated by many particle interactions are not known. We access these systems by a generalized cluster expansion, the photon-probability-cluster-expansion: a reliable approach for few photon dynamics in many body electron systems. As a first application, we discuss vacuum Rabi flopping and show that their amplitude determines the number of electrons in the quantum dot.
\end{abstract}

PACS numbers: 42.50.Ar,78.67.Hc

Introduction: For describing the quantum dynamics of semiconductor light emitters involving weak light-matter coupling and large photon numbers, an expansion involving mean field quantities and their fluctuations (often called cluster or correlation expansion) provides a well controlled theoretical scheme to treat many particle systems of electrons, phonons and photons [1, 2, 3, 4]. However, for semiconductor emitters operated in the limit of few photons [5] and few electronic levels, such as quantum dot-wetting layer (QD-WL) systems (cf. Fig. [1)) [4], the cluster expansion (as illustrated below) breaks down, since it is based on the assumption that fluctuations of mean field quantities have minor influence. With the rise of quantum information and the search for proper solid state emitters, QD-WL systems for single and entangled photons are in the focus of current research [3, 4, 6] . Therefore, there is an urgent need for the extension of the standard cluster expansion. Such an extension - the photon probability cluster expansion (PPCE) is developed in this Letter.

We generalize the cluster expansion [2, 3, 44] to the limit of few photons in systems with few quantum confined electronic levels such as semiconductor QD-WL systems, cf. Fig. 19). The strength of our method is also to keep the accurate results of the standard cluster expansion for large photon numbers [2, 3, 4] and the strong coupling
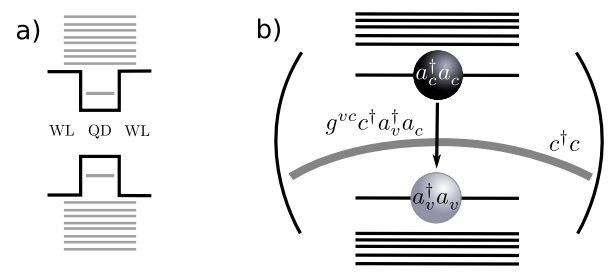

Figure 1: Scheme of the model system: a) a QD is embedded inside a WL leading to confined QD states coupled to a WL continuum, b) the emission of the confined states couples to a photon mode in a cavity. limit for high quality cavities [7].

To illustrate the standard cluster expansion and its break-down, we introduce $c^{\dagger}, c$ as the Bosonic construction/destruction operators for a single photon mode with frequency $\omega_{0}$ and $a^{\dagger}, a$ as the Fermionic operators for electrons. Subscripts $c, v$, denote the lowest confined conduction and valence band state of the $\mathrm{QD}$, respectively. The corresponding model of the QD-WL system is depicted in Fig. 1 b). In the following, this model will be used to illustrate our approach to reproduce exactly the Jaynes-Cummings model (JCM) (one electron approximation in atomic two level systems (i)) and extent it to many electron systems (QD-WL systems (ii)). To start, we illustrate the difference between the single and many electrons systems: The JaynesCummings model (JCM) 8], containing only one electron in an atomic two level system is exactly solvable by expanding the solution into states having distinct photon number and electronic state. Therefore, quantities of interest such as photon number $\left\langle c^{\dagger} c\right\rangle$, photon-photon correlation $\left\langle c^{\dagger} c^{\dagger} c c\right\rangle$, electronic densities $\left\langle a_{v}^{\dagger} a_{v}\right\rangle,\left\langle a_{c}^{\dagger} a_{c}\right\rangle$ can be calculated exactly. Here, the expectation value is denoted by $\langle\ldots\rangle=\operatorname{tr}(\ldots \rho)$, where $\rho$ is the statistical operator. In particular, by the exact expression $\left\langle a_{c}^{\dagger} a_{v} a_{v}^{\dagger} a_{c}\right\rangle=\left\langle a_{c}^{\dagger} a_{c}\right\rangle$ for a single electron, the hierarchy of quantum correlations is closed[9]. Unfortunately, however the JCM does not provide a scheme to include corrections resulting from further fermionic particles beyond the two level, one electron approximation. Beyond the JCM, i.e. in many electron systems, such as an occupied WL, Fig. 1, non-factorizing correlations such as $\left\langle a_{c}^{\dagger} a_{v} a_{v}^{\dagger} a_{c}\right\rangle^{c}=\left\langle a_{c}^{\dagger} a_{v} a_{v}^{\dagger} a_{c}\right\rangle-\left\langle a_{c}^{\dagger} a_{c}\right\rangle \neq 0$ are important 4, 10]. This is the case in particular for QDs embedded in a WL 4], since in this case the electrons in the WL and in the QD are Coulomb coupled: Electrons are scattered from the WL to QDs and vice versa, which lead to a correlation between electrons in the QDs and in WL[4]. The first correlation contribution requires an electron and a hole to be combined for emission. 
So far the standard cluster expansion of $\left\langle a_{c}^{\dagger} a_{v} a_{v}^{\dagger} a_{c}\right\rangle^{c}$ provides access only to large photon number dynamics or in weak coupling-short time limit $\left(\hbar\left|g^{v c}\right| \cdot t \ll 1\right.$, where $g^{v c}$ is the light matter coupling constant, see below). This is, because the standard cluster expansion constitutes a hierarchy of equations for correlations, in which the dynamics of relevant observables are not connected directly to the photon number state. Examples include quantities such as $\left\langle a_{c}^{\dagger} a_{c} c^{\dagger} c\right\rangle$, which include contribution from photon states with one and more photons. This will be illustrated later using Eq. (5), it is clear that this kind of expansion is not suitable, if correlations with a distinct number of photons are of crucial importance.

A theoretical scheme, which attacks this problem for a single photon mode, but is easy to extend to multiple photon modes, is presented in this Letter. This new approach will allow to treat few photon dynamics for quantum information in many particle systems such as QDWL systems with few electronic levels, that contribute to the emission.

Model system: We illustrate the proposed photonprobability cluster expansion (PPCE) using the basic light-matter Hamiltonian $H=H_{0}+H_{e l-p t}[9]$ :

$$
\begin{aligned}
H_{0} & =\hbar \omega_{0} c^{\dagger} c+\hbar \varepsilon_{c} a_{c}^{\dagger} a_{c}+\hbar \varepsilon_{v} a_{v}^{\dagger} a_{v} \\
H_{e l-p t} & =-\hbar\left(g^{v c} a_{v}^{\dagger} a_{c} c^{\dagger}+g^{v c *} a_{c}^{\dagger} a_{v} c\right),
\end{aligned}
$$

(illustrated in Fig1b)). For simplicity, we consider only a valence band $v$ and conduction band $c$ state with the energies $\hbar \varepsilon_{v}$ and $\hbar \varepsilon_{c}$ respectively. The off-diagonal coupling matrix $g^{v c}$ denotes the electron-photon-interaction strength. Additionally, the two level QD is assumed to be embedded, in an electronically occupied WL. In such a many particle system the JCM-valid factorization $\left\langle a_{v}^{\dagger} a_{c} a_{c}^{\dagger} a_{v}\right\rangle=\left\langle a_{v}^{\dagger} a_{v}\right\rangle$ has to be replaced by 2, [4] $\left\langle a_{v}^{\dagger} a_{c} a_{c}^{\dagger} a_{v}\right\rangle=\left\langle a_{v}^{\dagger} a_{v}\right\rangle\left(1-\left\langle a_{c}^{\dagger} a_{c}\right\rangle\right)+\left\langle a_{v}^{\dagger} a_{c} a_{c}^{\dagger} a_{v}\right\rangle^{\tilde{c}}$ [14] Clearly, because the one electron assumption in the QDWL system fails, Pauli-blocking terms are introduced [4]. Since the correlation $\left\langle a_{v}^{\dagger} a_{c} a_{c}^{\dagger} a_{v}\right\rangle^{\tilde{c}}$ cannot be treated exactly, an analytical solution of the model is not possible anymore, and the cluster expansion of $\left\langle a_{v}^{\dagger} a_{c} a_{c}^{\dagger} a_{v}\right\rangle$ provides only a perturbational approach.

We start showing that the cluster expansion yields unphysical behaviour in the few photon strong coupling limit for two electronic levels: Up to the order $\left|g^{v c}\right|^{2}$, using the Heisenberg equation of motion, the cluster expansion reads [3, 4] :

$$
\begin{aligned}
\partial_{t}\left\langle c^{\dagger} c\right\rangle= & -\partial_{t} f^{e}=-\partial_{t} f^{h}=-2 \operatorname{Im}\left(g^{v c *}\left\langle a_{v}^{\dagger} a_{c} c^{\dagger}\right\rangle\langle 3)\right. \\
\partial_{t}\left\langle a_{v}^{\dagger} a_{c} c^{\dagger}\right\rangle= & \imath\left(\varepsilon_{v}-\varepsilon_{c}+\omega_{0}+\imath \gamma_{p}\right)\left\langle a_{v}^{\dagger} a_{c} c^{\dagger}\right\rangle-\imath g^{v c *} f_{s p}^{e} \\
& -\imath g^{v c *}\left\langle c^{\dagger} c\right\rangle\left(f^{e}+f^{h}-1\right) .
\end{aligned}
$$

Here, we introduced the hole occupation $f^{h}=1-\left\langle a_{v}^{\dagger} a_{v}\right\rangle$, the electron occupation $f^{e}=\left\langle a_{c}^{\dagger} a_{c}\right\rangle$ and the phenomenological pure dephasing constant $\gamma_{p}$. The spontaneous emission term $f_{s p}^{e}$ is equal to $f^{e}$ in case of the JCM and

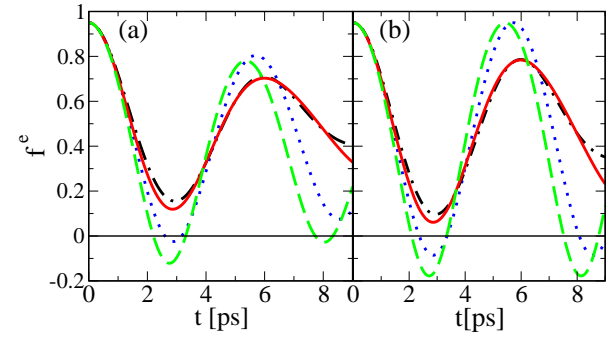

Figure 2: The JCM (solid/red), standard cluster-expansion atom like using one electron assumption (dashed/green), and standard cluster-expansion using $f_{s p}^{e}=f^{e} f^{h}$ in QD-WL system (dotted/blue) and PPCE using $f_{s p, n}^{e}=f_{n}^{e} f_{n}^{h}$ in QD-WL system in new scheme (dotted dashed/black). With the initial conditions: $f^{e}=f^{h}=0.95, p_{0}=0.8, p_{1}=0.2$ and a coupling constant $\left|g^{v c}\right|=0.51 /$ fs for a QD resonant with the cavity mode. ( (a) $\gamma_{p}=1 /(10 \mathrm{ps})$ and $\left.(\mathrm{b}) \gamma_{p}=0 /(\mathrm{ps})\right)$

$f_{s p}^{e}=\left\langle a_{v}^{\dagger} a_{c} a_{c}^{\dagger} a_{v}\right\rangle=f^{e} f^{h}$ for the QD-WL case [4]. In Fig. 2 we plot the dynamics of the electron occupation for: (i) exact JCM model [8], (ii) cluster-expansion using one electron assumption $f_{s p}^{e}=f^{e}$, (iii) for the QD-WL system $f_{s p}^{e}=f^{e} f^{h}$ and also (iv) for the below developed PPCE, introduced later.

The exact JCM solution for the one electron assumption leads to well known vacuum Rabi flops. Fig. 2 shows clearly that the standard cluster expansion of the JCM and the QD-WL system lead to negative and unphysical results in the few photon limit. However, we note that in the many QD and/or many photon case $\left\langle c^{\dagger} c\right\rangle \gg 1$, correlation expansion provides an excellent tool [4, 10]. As expected, all approaches agree for small times (weak coupling limit $\left.\hbar^{-1}\left|g^{v c}\right| t \ll 1\right)$.

The breakdown of the cluster expansion results from the fact that the system is completely characterized with mean single particle quantities. This is problematic in the limit of few electrons and photons, since correlations (in particular fluctuations) between electrons and photon number states are crucial in this limit. To improve this situation, we focus now on the development of the PPCE.

Photon-Probability Cluster Expansion (PPCE): Important quantities to characterize a quantum optical field are the normalized intensity-intensity correlation function $g^{(2)}(\tau=0)=\frac{\left\langle c^{\dagger} c^{\dagger} c\right\rangle}{\left\langle c^{\dagger} c\right\rangle^{2}}$ and the photon intensity $g^{(1)}(\tau=0)=\left\langle c^{\dagger} c\right\rangle[9]$. Both quantities can be measured and they are directly connected to the photon number $\left\langle c^{\dagger} c\right\rangle$ and intensity-intensity expectation value $\left\langle c^{\dagger} c^{\dagger} c c\right\rangle$. The idea - to circumvent the problems characteristic for the cluster expansion (cf. Fig. 2) - is to formulate all quantities in terms of $n$ photon probability $p_{n}=\langle\mid n\rangle\langle n \mid\rangle$, where $|n\rangle$ denotes the Fock-state of $n$ photons in the system. This idea is already succesfully applied in JCM [8], but needs to be generalized to the many electron case. The PPCE introduced now combines the advantages of the exact JCM-solution and the standard cluster expan- 
sion. The observables read in the Fock basis:

$$
\left\langle c^{\dagger} c\right\rangle=\sum_{n=1}^{\infty} n p_{n},\left\langle c^{\dagger} c^{\dagger} c c\right\rangle=\sum_{n=2}^{\infty} n(n-1) p_{n} .
$$

Eq. (5) show that it is sufficient to calculate the equations of motion for the probability to find $n$ photons $p_{n}=\langle\mid n\rangle\langle n \mid\rangle$ in the system. Heisenberg equations for $p_{n}$, using the Hamiltonian, Eqs. (112), couple to assisted occupation probabilities: $\langle\mid n\rangle\left\langle n \mid a_{c}^{\dagger} a_{c}\right\rangle,\langle\mid n\rangle\left\langle n \mid a_{v}^{\dagger} a_{v}\right\rangle$ and transitions $\langle\mid n\rangle\left\langle n+1 \mid a_{c}^{\dagger} a_{v}\right\rangle$ or similar quantities if a more general system is considered. We discuss the application of the theory to the JCM (one electron) and the QD-WLsystem (many electrons) separately:

For the case of one electron (JCM case), the equation of motion for the probability $p_{n}$ reads:

$$
\begin{aligned}
\partial_{t} p_{n}= & -2 \sqrt{n} \operatorname{Im}\left(g^{v c}\langle\mid n\rangle\left\langle n-1 \mid a_{v}^{\dagger} a_{c}\right\rangle\right) \\
& +2 \sqrt{n+1} \operatorname{Im}\left(g^{v c}\langle\mid n+1\rangle\left\langle n \mid a_{v}^{\dagger} a_{c}\right\rangle\right) .
\end{aligned}
$$

The $n$-photon probability $p_{n}$ is driven by the photon transition assisted polarization $\langle\mid n+1\rangle\left\langle n \mid a_{v}^{\dagger} a_{c}\right\rangle$ :

$$
\begin{aligned}
\partial_{t} & \langle\mid n+1\rangle\left\langle n \mid a_{v}^{\dagger} a_{c}\right\rangle \\
& =\imath\left(\varepsilon_{v}-\varepsilon_{c}+\omega_{0}+\imath \gamma_{p}\right)\langle\mid n+1\rangle\left\langle n \mid a_{v}^{\dagger} a_{c}\right\rangle \\
& +\imath g^{v c *} \sqrt{n+1}\left(p_{n+1}-f_{n+1}^{h}\right)-\imath g^{v c *} \sqrt{n+1} f_{n}^{e} .(7)
\end{aligned}
$$

Besides the free energy rotation quantum optical absorption and emission processes occur in the last line. These processes are related to $f_{n}^{h}=p_{n}-\langle\mid n\rangle\left\langle n \mid a_{v}^{\dagger} a_{v}\right\rangle$ and $f_{n}^{e}=\langle\mid n\rangle\left\langle n \mid a_{c}^{\dagger} a_{c}\right\rangle$ for hole and electron densities assisted by $n$ photons. Finally, the electron and hole densities $f_{n}^{e / h}$ couple to quantum optical transitions:

$$
\partial_{t} f_{n}^{e / h}=2 \sqrt{n+1} \operatorname{Im}\left(g^{v c}\langle\mid n+1\rangle\left\langle n \mid a_{v}^{\dagger} a_{c}\right\rangle\right) .
$$

To close the hierarchy of equations of motion we used $\left\langle a_{i}^{\dagger} a_{j}^{\dagger} a_{k} a_{l} \mid n\right\rangle\langle n \mid\rangle=0$ strictly valid only for the single electron case, since two electrons are annihilated. Eqs. (668) without pure dephasing reproduces the JCM [8]. This is an important benchmark of the new PPCE.

If we consider more than one electron ( $Q D$-WL case), $\partial_{t}\langle\mid n+1\rangle\left\langle n \mid a_{v}^{\dagger} a_{c}\right\rangle$, couples to $\left\langle a_{v}^{\dagger} a_{c}^{\dagger} a_{c} a_{v} \mid n\right\rangle\langle n \mid\rangle \neq 0$. In order to truncate the PPCE hierarchy of equations of motion at least approximately, we need factorization rules for correlation between electrons, but also for other bosons to e.g. introduce cavity loss and radiative decay consistently.

We use and generalize two basic factorization limits, Hartree-Fock and Born approximation (i,ii), respectively.

(i) The Hartree-Fock approximation $\left\langle a_{i}^{\dagger} a_{j}^{\dagger} a_{k} a_{l}\right\rangle \approx$ $\left\langle a_{i}^{\dagger} a_{l}\right\rangle\left\langle a_{j}^{\dagger} a_{k}\right\rangle-\left\langle a_{i}^{\dagger} a_{k}\right\rangle\left\langle a_{j}^{\dagger} a_{l}\right\rangle$ is based on the assumption [1, 12], that the density matrix $\rho$ can be described by single particle quantities using the Generalized Canonical Statistical Operator (GCSO) $\rho(t) \approx \exp \left[-\sum_{i j} \lambda_{i j}(t) a_{i}^{\dagger} a_{j} /\left(k_{B} T\right)\right] / Z[12]$ with the Lagrange parameters $\lambda_{i j}(t)$. For the $\mathrm{n}$-photon number contribution of the density matrix $\langle n|\rho(t)| n\rangle / p_{n}$, a photon number dependence of the Lagrange parameters is introduced: $\langle n|\rho(t)| n\rangle / p_{n} \approx \exp \left[-\sum_{i j} \lambda_{i j}^{n}(t) a_{i}^{\dagger} a_{j} /\left(k_{B} T\right)\right] / Z$. From this, we recognize the factorization rule: $\langle\mid n\rangle\left\langle n \mid a_{1}^{\dagger} a_{2}^{\dagger} a_{3} a_{4}\right\rangle=\left(\langle\mid n\rangle\left\langle n \mid a_{1}^{\dagger} a_{4}\right\rangle\langle\mid n\rangle\left\langle n \mid a_{2}^{\dagger} a_{3}\right\rangle-\right.$ $\left.\langle\mid n\rangle\left\langle n \mid a_{1}^{\dagger} a_{3}\right\rangle\langle\mid n\rangle\left\langle n \mid a_{2}^{\dagger} a_{4}\right\rangle\right) / p_{n}$.

(ii) The second order-Born approximation for a correlated electron-photon system embedded in a bosonic bath (e.g. phonons or other photon modes $b^{\dagger}, b$ ) assumes that the density matrix can be factorized: $\rho \approx \rho_{B} \otimes \rho_{e l-p t}$, which implies: $\langle\mid n\rangle\left\langle n \mid a_{1}^{\dagger} a_{2} b_{3}^{\dagger} b_{4}\right\rangle \approx\langle\mid n\rangle\left\langle n \mid a_{1}^{\dagger} a_{2}\right\rangle\left\langle b_{3}^{\dagger} b_{4}\right\rangle$. In particular, for QD-WL system, to some electronic states bath like properties (e.g. WL states) can be attributed, so a factorizing density matrix $\rho=\rho_{\text {el-uncorr }} \otimes \rho_{p h+c o r r}$ can be assumed. This leads to a factorization rule: $\langle\mid n\rangle\left\langle n \mid a_{i}^{\dagger} a_{j}\right\rangle \approx\langle\mid n\rangle\langle n \mid\rangle\left\langle a_{i}^{\dagger} a_{j}\right\rangle$ for WL states $i, j$.

Our factorization rules can be extended to include high order PPCE correlations, preserving the important electron-photon number correlations. We now evaluate the equations of motion in the PPCE approach: In comparison to the single electron case, the equation for $p_{n}$ (Eq. (6)) does not change. Eq. (7) is modified for the QD-WL system case using the modified Hartree-Fock factorization rule:

$$
\begin{aligned}
\partial_{t} & \langle\mid n+1\rangle\left\langle n \mid a_{v}^{\dagger} a_{c}\right\rangle \\
& =\imath\left(\varepsilon_{v}-\varepsilon_{c}+\omega_{0}+\imath \gamma_{p}\right)\langle\mid n+1\rangle\left\langle n \mid a_{v}^{\dagger} a_{c}\right\rangle \\
& +\imath g^{v c} \sqrt{n+1}\left(p_{n+1}-f_{n+1}^{h}\right)\left(p_{n+1}-f_{n+1}^{e}\right) / p_{n+1} \\
& -\imath g^{v c \sqrt{n+1}} f_{n}^{h} f_{n}^{e} / p_{n} .
\end{aligned}
$$

In comparison to the one electron assumption, Eq. (7) the terms $\left(p_{n+1}-f_{n+1}^{h}\right)$ and $f_{n}^{e}$ are replaced with $\left(p_{n+1}-\right.$ $\left.f_{n+1}^{h}\right)\left(p_{n+1}-f_{n+1}^{e}\right) / p_{n+1}$ and $f_{n}^{h} f_{n}^{e} / p_{n}$ respectively. Similarly, Eq. (8) does not change. (In Eq. (9), some sourceless terms including $\langle\mid n\rangle\left\langle n \mid a_{v}^{\dagger} a_{c}\right\rangle$ are neglected.) Eqs. (6), (8), (91) constitute the new quantum optical dynamics for a few photon/ few electronic level system.

Discussion: The best benchmark for the new PPCE scheme is the case of vacuum Rabi flops where quantum fluctuations dominate the dynamics. For strong coupling, the in-scattering rate from the WL has to be insignificant compared to $g^{v c}$. Ref. 11 suggest for $g^{v c}$ chosen here, WL densities $\ll 10^{11} \mathrm{~cm}^{-2}$. Now, we reiterate our discussion of Fig. 2. Obviously, only the PPCE (dotted dashed) provides physical reasonable results as opposed to the standard cluster expansion method, since it guarantees positivity of the electronic density. In particular, we recover the exact JCM solution. The Pauli blocking term occuring in the QD-WL system does not lead to strong deviations for not long times from the JCM case. For the calculations in Fig. 2, we assumed, that initially there is one electron $\left(f^{e}+\left(1-f^{h}\right)\right)=1$ in the $\mathrm{QD} f^{h}=f^{e}$. For the numerically evaluation, we choose 

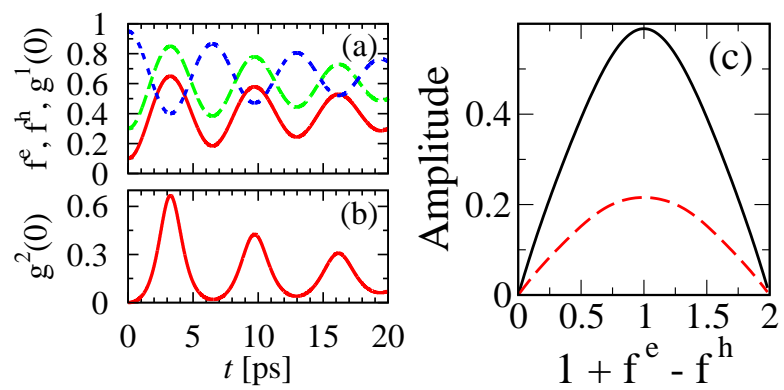

Figure 3: The PPCE solution for semiconductor QDs. With the initial conditions: $f^{h}=0.3$ and $f^{e}=0.1, p_{0}=0.05$, $p_{1}=0.95$ and a coupling constant $g=0.5 \mathrm{meV}$ for a QD resonant with the cavity mode and $\gamma_{p}=(10 \mathrm{ps})^{-1}$. In (a) the different lines are $f^{e}$ (solid/red), $f^{h}$ (green/long dashed), $g^{1}(0)$ (blue/short dashed). (c) The maximum value of $g^{(2)}$ for PPCE is plotted over the number of electrons in the QD, with $p_{1}=1.0$ (black/straight) and $p_{2}=1.0$ (red/dashed).

as initial correlations a finite photon number distribution $p_{n}\left(t_{0}\right)$, whereas the initial transitions $\langle\mid n+1\rangle\left\langle n \mid a_{v}^{\dagger} a_{c}\right\rangle$ will be set to zero, i.e. assuming an uncorrelated initial state. Also vanishing initial correlations between electron and hole system are assumed: The photon number, electron and hole densities will be set to $f_{n}^{h}=f^{h} \cdot p_{n}\left(t_{0}\right)$ and $f_{n}^{e}=f^{e} \cdot p_{n}\left(t_{0}\right)$. Comparing the PPCE calculation to the cluster expansion and the JCM, only the PPCE recovers the exact JCM result. Therefore, we assume that it is more valid for the QD-WL case than the standard cluster expansion. The damping $\gamma_{p}$ of Rabi flopping describes dephasing due to electron-phonon coupling and Coulomb correlations betweeen QD and WL [11].

We now focus on the specific QD-WL properties. In Fig. 3 the dynamics of $f^{e}, f^{h}, g^{(1)}$ and $g^{(2)}$ is plotted for semiconductor specific initial values $f^{h} \neq f^{e}$, occuring in QD-WL systems [4]. The average photon number oscillates between 0 and 1 , the $g^{(2)}$ between 0 and 0.7 is in the anti bunching regime [9]. After the first Rabi flop the system goes into the single photon emitter regime with a $g^{(2)}<0.5[13$. In contrast to the JCM, the strength of the vacuum Rabi flopping is reduced in dependence on how strong the electron and hole density deviates from the one electron assumption $f^{h}=f^{e}$. This implies that the amplitude of the Rabi flops might be used as a measure for the number of electrons in the actual $Q D$. Since the amplitude of the Rabi flopping is directly connected to $g^{(2)}$, it may be possible to stear $g^{(2)}$ with the deviation from the one electron assumption. This is illustrated more clearly in Fig. 35), where the number of electrons in the QD is plotted as a function of the maximum of $g^{(2)}$, which is directly connected to the maximum of vacuum Rabi flopping. It is seen that a maximum of $g^{(2)}$ is achieved, if we have only one electron $f_{e}=f_{h}$. This behaviour can be attributed to the Pauli-blocking terms $f_{e}^{n} \cdot f_{h}^{n}$, because they drive the emission and are enhanced in the one electron case.
In conclusion: a modified cluster expansion scheme to describe, semiconductor quantum dot - wetting layer quantum optical devices in the strong coupling limit involving few photons and few dominant electronic states is introduced. This photon-probability-cluster expansion (PPCE), reproduces the Jaynes-Cummings model (JCM) as a well known benchmark in quantum electrodynamics, the standard cluster expansion cannot. For the QD-WL system, we have shown that the amplitude of vacuum Rabi flopping and the maximum of $g^{(2)}$ depend on the numbers of electron and holes pumped into the quantum dot device. This is a first important prediction from the PPCE.

We envision, that the new scheme can be applied to many solid state structures with few electronic states limit in the few photon limit. The approach will stimulate further investigation beyond JCM: Our approach allows to include electron-phonon interaction and Coulomb interaction additionally to the already demonstrated electron-photon interaction in a similar fashion.

We acknowledge support from Deutsche Forschungsgemeinschaft through SFB 787 and A.S. acknowledges financial support from the DAAD.

* marten.richter@tu-berlin.de

[1] M. Lindberg and S. W. Koch, Phys. Rev. B 38, 3342 (1988) , J. Fricke, Ann. Phys. 252, 479 (1996), F. Rossi and T. Kuhn, Rev. Mod. Phys. 74, 895 (2002), P. Machnikowski and L. Jacak, Phys. Rev. B 69, 193302 (2004).

[2] M. Kira, F. Jahnke, W. Hoyer, and S. W. Koch, Prog. Quantum Electron. 23, 189 (1999), M. Kira and S. Koch, Prog. Quantum Electron. 30, 155 (2006).

[3] K. J. Ahn, J. Förstner, and A. Knorr, Phys. Rev. B 71, 153309 (2005), A. Carmele, A. Knorr, and M. Richter, Phys. Rev. B 79, 035316 (2009).

[4] N. Baer, et al. , Eur. Phys. J. B 50, 411 (2006), C. Gies, et al. , Phys Rev A 75, 013803 (2007), C. Gies, J. Wiersig, and F. Jahnke, Phys. Rev. Lett. 101, 067401 (2008).

[5] P. Michler et al. , Nature 406, 968 (2000), D. Press, et al. , Phys. Rev. Lett. 98, 117402 (2007).

[6] Z. Yuan, et al. , Science 295, 102 (2002) , T. Yoshie, et al., Nature 432, 200 (2004), C. Böckler, et al., Appl. Phys. Lett. 92, 091107 (2008).

[7] J. P. Reithmaier,et al. , Nature 432, 197 (2004).

[8] E. Jaynes and F. Cummings, Proc.IEEE 51, 89 (1963).

[9] L. Mandel and E. Wolf, Optical coherence and quantum optics (Cambridge University Press, Cambridge, 1995).

[10] L. Schneebeli, M. Kira, and S. W. Koch, Phys. Rev. Lett. 101, 097401 (2008).

[11] T. R. Nielsen, P. Gartner, and F. Jahnke, Phys. Rev. B 69, 235314 (2004).

[12] E. Fick and G. Sauermann, The Quantum Statistics of Dynamic Processes (Springer, Berlin, 1990).

[13] B. Lounis and M. Orrit, Rep. Prog. Phys. 68, 1129 (2005).

[14] The on the index $c$ is used to distingish this correlation from the earlier introduced $\left\langle a_{c}^{\dagger} a_{v} a_{v}^{\dagger} a_{c}\right\rangle^{c}$. 